\title{
Effets de la saison de vêlage et de quelques paramètres zootechniques sur la production laitière dans les hauts plateaux de l'ouest du Cameroun
}

\author{
P. Kamga ${ }^{1}$ J.N. Mbanya ${ }^{1}$ N.R. Awah ${ }^{1}$ \\ Y. Mbohou ${ }^{2}$ Y. Manjeli ${ }^{2}$ A. Nguemdjom ${ }^{1}$ \\ B. Kamga Pamela ${ }^{1}$ R.M. Njwe ${ }^{1}$ P.H. Bayemi ${ }^{1}$ \\ C. Ndi ${ }^{1}$ H. Imélé ${ }^{1}$ A. Kameni ${ }^{1}$
}

\section{Mots-clés}

Bovin - Croisement - Production laitière - Intervalle entre parturitions Facteur lié au site - Numéro de lactation - Cameroun.

\begin{abstract}
Résumé
Des données sur 275 lactations collectées entre 1981 et 1991 ont été analysées. Le but de ce projet a été d'étudier l'effet de la saison de vêlage, de quelques paramètres zootechniques, de l'intervalle entre vêlages des races Holstein et Jersiaise et de leurs croisements avec le zébu Goudali et le zébu Peul blanc (White Fulani) sur la production laitière dans les hauts plateaux de I'ouest du Cameroun. Les résultats suivants ont été obtenus :

- la production laitière des races pures Holstein $(n=73)$ et Jersiaise $(n=32)$, sans être comparable à celle de leur pays d'origine, a été significativement supérieure à celle des autres types génétiques étudiés, la Holstein ayant été plus performante que la Jersiaise ( $2508 \pm 105$ I en $291 \pm 10$ jours, contre $1818 \pm 137$ I en $251 \pm 13$ jours) ;

- en croisement de première génération avec le zébu local, la Holstein $(n=52)$ a produit significativement plus de lait que la Jersiaise $(n=57)$. La Holstein $x$ le zébu Goudali a produit plus de lait que la Jersiaise x le zébu Peul blanc ( $1940 \pm 109$ I en $270 \pm 10$ jours, contre $1550 \pm 106$ I en $257 \pm 10$ jours). La production laitière des races croisées trois quarts Jersiaises ( $n=61)$ a été plus élevée ( $1774 \pm 117$ en $250 \pm 11$ jours) ;

- I'année et le numéro de lactation ont influencé significativement la production laitière et la durée de lactation, alors que la saison de vêlage n'a pas eu d'effet significatif sur ces deux paramètres.

L'analyse financière et les recherches sur la composition du lait devraient se poursuivre pour des recommandations plus concrètes.
\end{abstract}

\section{INTRODUCTION}

Le recours au croisement des races indigènes zébus (Bos indicus) avec les races exotiques taurines (Bos taurus) est très souvent envisagé pour améliorer les performances des races locales généralement caractérisées par leur faible productivité. Cette méthode très délicate et difficile à mener à bien vise, par apport d'un sang nouveau, à accroître rapidement la taille et le poids, le niveau qualitatif

1. Irad Bambui, PO Box 51, Bamenda, Cameroun ; tél. : +237 756051

2. Université de Dschang, PO Box 110, Dschang, Cameroun et la capacité moyenne de production de lait, de viande, ou à apporter des qualités nouvelles. Les spécialistes en productions animales peuvent obtenir une meilleure productivité des races locales par l'utilisation de diverses méthodes de croisement : croisement industriel avec des races très précoces (métis de première génération livrés à la boucherie), croisement de retrempe (introduction d'un reproducteur de race différente une seule fois pour ramener une qualité qui tend à disparaître), croisement d'absorption (substitution à la race locale d'une race étrangère plus productive que le troupeau initial). Une telle amélioration requiert, outre des conditions d'élevage rationnelles (habitat et santé), des conditions favorables d'alimentation et une bonne organisation d'élevage. Auparavant, l'introduction 
d'animaux exotiques pour accroître la productivité des races locales a souvent échoué dans les zones tropicales en raison de l'inadaptation des métis aux conditions environnementales prévalentes.

Au Cameroun, l'importation des races bovines exotiques date de l'époque coloniale et, plus récemment, les races Holstein et Jersiaise ont été importées des Etats-Unis d'Amérique en 1974 par l'Institut de recherches zootechniques pour l'évaluation de leurs performances et celles de leurs croisements avec les races locales. Des études préliminaires amorcées par Mbah et coll. (12) montrent que les vaches de race pure produisent plus de lait que leurs croisements avec le zébu local. Or, depuis 1984, l'Institut de recherches zootechniques s'efforce d'amortir l'effet de la saison sèche sur les vaches laitières grâce à une production régulière d'ensilage (7).

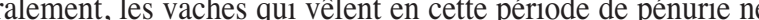
trouvent pas toujours suffisamment d'herbe pour couvrir leurs besoins d'entretien et de production.

Dans ce projet ont été étudiés les effets de la saison, de l'année de vêlage et de quelques paramètres zootechniques sur la production laitière des races Holstein et Jersiaise et de leurs croisements (avec le zébu Goudali et le zébu Peul blanc) à la station de l'Institut de recherches agricoles pour le développement (Irad) de Bambui dans les hauts plateaux de l'ouest du Cameroun.

\section{MATERIEL ET METHODES}

\section{Caractéristiques géoclimatiques du site}

L'altitude varie entre 1600 et $1982 \mathrm{~m}$. Le climat de cette région est camerounais d'altitude, à trois saisons : une saison sèche de novembre à février, une saison des pluies précoces de mars à juin et une saison des pluies tardives de juillet à octobre. Les précipitations mensuelles moyennes enregistrées à la station météorologique de Bambui pendant ces périodes ont été respectivement de $31,7 \mathrm{~mm}, 209,5 \mathrm{~mm}$ et $351,4 \mathrm{~mm}$, avec une précipitation annuelle de $2370 \mathrm{~mm}$. L'humidité relative moyenne a varié entre 52 p. 100 en saison sèche et 79 p. 100 en saison des pluies. Les températures minimales ont été comprises entre 14,2 et $16,6^{\circ} \mathrm{C}$ et les températures maximales ont fluctué entre 21,9 et $27,1^{\circ} \mathrm{C}$. La végétation est de type savane arbustive avec des galeries-forestières par endroits. Les maladies les plus répandues sont causées par les tiques et les parasites internes.

\section{Pâturages}

Le couvert végétal est constitué de graminées fourragères introduites (Brachiaria ruziziensis, Tripsacum laxum) et d'espèces spontanées comme Pennisetum purpureum, Pennisetum clandestinum et Sporobolus africanus. Les pâturages, généralement envahis par la fougère aigle (Pteridium aquilinum), sont divisés en parcs délimités par des rideaux d'eucalyptus et d'Erythrina poeppigiana.

\section{Conservation des fourrages}

L'ensilage de Tripsacum laxum et de Pennisetum purpureum a été fabriqué et distribué à volonté sur la presque totalité des saisons sèches de la période de l'étude. De même, le fourrage de Sporobolus africanus et de Brachiaria spp. a été utilisé régulièrement au cours des années de l'étude.

\section{Bâtiments d'élevage}

Les bâtiments d'élevage ont compris :

- l'aire d'alimentation (étable d'affouragement où les vaches ont reçu en saison sèche du fourrage, du concentré et de l'ensilage et en saison des pluies du concentré seulement);
- l'atelier de préparation des aliments, salle où des ingrédients divers, comme le son de riz, le maïs, le tourteau de coton, la poudre d'os et le sel de cuisine, ont souvent été mélangés pour l'alimentation des animaux ;

- la salle de traite, équipée d'une machine à traire mécanique pour la récolte du lait ;

- la maternité où les vaches ont été transférées à l'approche de la mise bas ;

- le logement des veaux dans des boxes de 1,5 x 2 m chacun, équipés d'un râtelier, d'une mangeoire et d'un abreuvoir ;

- la salle de soins où les animaux peuvent être traités (vaccination, injection...) ou mis en quarantaine.

\section{Animaux}

Deux groupes génétiques ont composé le troupeau, les vaches Holstein et Jersiaise. Les vaches croisées $1 / 2$ Holstein x 1/2 zébu Goudali, $1 / 2$ Jersiaise x $1 / 2$ zébu Peul blanc, 3/4 Jersiaise x 1/4 zébu Peul blanc étaient des métisses obtenues en station par insémination artificielle et en monte naturelle.

\section{Alimentation des vaches}

Pendant la saison des pluies (mars à novembre), les bergers ont fait paître les vaches en rotation dans les parcs de 9 à $16 \mathrm{~h}$. L'alimentation en concentré a consisté à administrer $2 \mathrm{~kg}$ à $7 \mathrm{~h}$ et $2 \mathrm{~kg}$ à $16 \mathrm{~h}$ avant la traite.

En saison sèche (novembre à mars), les vaches ont été nourries sur ensilage d'herbe de Guatemala et d'herbe à éléphant. Elles ont parfois également reçu du foin de Brachiaria ruziziensis ou de Sporobolus africanus. L'eau a été donnée ad libitum dans les abreuvoirs en béton placés dans les parcs ou à l'étable. Les vaches en lactation ont reçu chacune $4 \mathrm{~kg}$ d'un concentré composé de 38 p. 100 de maïs, 30 p. 100 de son de riz, 30 p. 100 de tourteau de coton, 1 p. 100 de poudre d'os et 1 p. 100 de sel de cuisine. La composition chimique calculée du concentré a montré un taux de 74,6 p. 100 de nutriments digestibles totaux et de 16 p. 100 de protéines.

\section{Alimentation des veaux}

Dès la mise bas, les veaux ont reçu du colostrum à volonté de leur mère pendant les quatre premiers jours. Ensuite, ils ont été séparés de celle-ci et placés dans des loges individuelles où ils y ont reçu chacun 2 à 41 de lait par jour selon leur poids à la naissance. Une semaine après la mise bas, le concentré a été introduit progressivement (jusqu'à $2 \mathrm{~kg}$ par jour), puis le fourrage a été servi ad libitum à deux semaines d'âge. L'abreuvement s'est fait à volonté.

\section{Protection sanitaire}

Les animaux ont été déparasités et vaccinés régulièrement contre la pasteurellose et le charbon bactéridien. Les animaux ont été déparasités une fois par semaine en saison sèche et deux fois par semaine en saison des pluies contre les tiques. Le parasitisme gastro-intestinal et pulmonaire des bovins sont des maladies communes dans la région. Des vermifuges au Panacur (fenbendazole) ont été administrés tous les deux mois. La lutte contre les tiques qui transmettent la cowdriose, la babésiose et l'anaplasmose s'est faite à l'aide du Supona (chrophenvinphos, organophosphoré), à raison de $0,5 \mathrm{ml}$ par $1000 \mathrm{ml}$ d'eau.

\section{Collecte des données}

La traite a été faite à l'aide d'une machine de marque Alfa Laval et le lait obtenu respectivement à 6 et $16 \mathrm{~h}$ a constitué la production du jour. La durée de lactation a été évaluée à partir de la collecte du lait après la mise bas jusqu'au tarissement. L'intervalle entre vêlages a représenté la durée enregistrée entre deux vêlages successifs. Pour chaque vache, le numéro d'identification, la date de 
mise bas, l'année, le numéro de la mise bas, la quantité de lait produite par jour et par lactation ont été enregistrés. En revanche, la composition du lait des vaches individuelles n'a pas été analysée, ce qui n'a pas permis de calculer les teneurs en matières grasses, en protéines et en matières sèches.

\section{Analyses statistiques}

Les données ont été analysées à l'aide du logiciel Systat selon les modèles ci-dessous.

Pour la production laitière et la durée de lactation,

$\mathrm{Y}_{\mathrm{ijkl}}=\mathrm{R}_{\mathrm{i}}+\mathrm{S}_{\mathrm{j}}+\mathrm{A}_{\mathrm{k}}+\mathrm{L}_{\mathrm{l}}+\mathrm{R} * \mathrm{~S}_{(\mathrm{ij})}+\mathrm{e}_{\mathrm{ijklm}}$, où

$\mathrm{Y}_{\mathrm{ijkl}}=$ production laitière ou durée de lactation observée sur chaque vache ;

$\mathrm{R}_{\mathrm{i}}=$ race $; \mathrm{i}=1 . . .5$;

$\mathrm{S}_{\mathrm{j}}=$ saison de vêlage $; \mathrm{j}=1 . . .3$;

$A_{k}=$ année de vêlage $; \mathrm{k}=1 \ldots 11$;

$\mathrm{L}_{1}=$ numéro de lactation $; \mathrm{l}=1 \ldots 8$;

$\mathrm{R} * \mathrm{~S}_{(\mathrm{ij})}=$ interaction entre race et saison.

Pour l'intervalle entre vêlages,

$\mathrm{Y}_{\mathrm{ijkl}}=$ intervalle entre vêlages observé sur chaque vache ;

$\mathrm{R}_{\mathrm{i}}=$ race $; \mathrm{i}=1 . . .4$;

$\mathrm{S}_{\mathrm{j}}=$ saison de vêlage $; \mathrm{j}=1 \ldots 3$;

$\mathrm{L}_{\mathrm{k}}=$ numéro de lactation, $; \mathrm{k}=1 \ldots 8$;

$\mathrm{R} * \mathrm{~S}_{(\mathrm{ij})}=$ interaction entre race et saison.

La méthode de Duncan a été utilisée pour comparer les moyennes quand elles étaient significativement différentes.

\section{RESULTATS ET DISCUSSION}

\section{Production laitière}

L'analyse de variance (tableau I) a montré une différence très significative $(P<0,01)$ entre les races, les années et les numéros de lactation pour ce paramètre. En revanche, aucune différence importante $(\mathrm{P}>0,05)$ n'a été observée entre les saisons de vêlage.

\section{Effet de la race sur la production laitière}

Pour la quantité de lait, la race Holstein a été significativement supérieure en race pure (2 508 l) et en croisement F1 (Holstein X
Goudali) (1 940 1) à tous les autres types génétiques étudiés. Ces résultats sont conformes à ceux trouvés par Mbah et coll. (12). La race pure Jersiaise vient ensuite avec 18181 . En revanche, chez les vaches croisées avec la Jersiaise, la production a augmenté proportionnellement quand le niveau d'absorption de la Jersiaise est passé de 50 à 75 p. 100. Les $1 / 2$ Jersiaise x $1 / 2$ zébu Peul blanc et 3/4 Jersiaise x 1/4 zébu Peul blanc ont eu une production moyenne respective de 1550 et 17741 . Les productions laitières moyennes sont présentées dans le tableau II en fonction de la race, de la saison, de l'année de vêlage et du numéro de lactation.

Les résultats obtenus ici confirment le haut potentiel des races Holstein et Jersiaise et surtout leur faculté d'adaptation en milieu tropical, même si les performances obtenues sont très inférieures à celles de leur pays d'origine [9 1281 de lait en 305 jours de lactation pour la Holstein aux Etats-Unis (22)]. En France, par exemple, les races Prim'Holstein et Jersiaise ont produit au contrôle laitier en 1997 respectivement $7342 \mathrm{~kg}$ en 310 jours et $4138 \mathrm{~kg}$ en 300 jours de lactation (6). Plusieurs facteurs environnementaux qui influencent la capacité de production sont connus et répertoriés. Il s'agit de problèmes pathologiques, de variations climatiques, etc. C'est ainsi que les performances varient en fonction du milieu d'élevage. Les résultats de cette étude ont été inférieurs aux 44711 pour la Holstein et 29891 pour la Jersiaise obtenus par Branton et coll. (2) aux EtatsUnis. Nos résultats s'expliquent par les variations de température qui ont parfois atteint $27^{\circ} \mathrm{C}$ affectant sensiblement la production et l'appétit chez les vaches (17). Pour la race Jersiaise, les résultats de cette étude ont été inférieurs à ceux de Duc et Taneja en Inde (2026 1) (4) et à ceux d'Adeniji (3 036 1) (1). En revanche, ils ont été comparables à ceux de Meyn et Wilkins (1 278 l) (14). Pour les vaches croisées Holstein, ils ont été proches de ceux de Prada (2 261 1) (17) mais inférieurs à ceux de Branton et coll. (2 502) (2) et Friestas et coll. (2 471 1) (5). De même, pour les vaches croisées $1 / 2$ Jersiaises, ils ont été inférieurs à ceux de Branton et coll. (2 360 1) (2) et comparables à ceux de Parmar et coll. (1 679 l) (15) mais supérieurs à ceux de Letenneur (1 332 1) en Côte d'Ivoire (9).

\section{Effet de la saison de vêlage sur la production laitière}

La saison de vêlage n'a pas eu d'effet significatif sur la production laitière. Cependant, d'une manière générale, les vaches qui ont vêlé en pleine saison des pluies ont eu tendance à produire plus de lait (1 991 1) que celles qui ont vêlé en saison sèche (1 893 l) et au début de la saison des pluies (1 865 l). Il semble que l'utilisation régulière d'ensilage et de concentrés a contribué à réduire le déficit alimentaire pendant la saison sèche (7). Kiwuwa et coll. ont trouvé des variations saisonnières semblables en Ethiopie (8).

\section{Tableau I}

Degrés de liberté $(\mathrm{DI})$, carrés moyens $(\mathrm{Cm})$ de la production laitière, de la durée de lactation et de l'intervalle entre vêlages en fonction de la saison, de l'année de vêlage et du numéro de lactation

\begin{tabular}{|c|c|c|c|c|c|c|}
\hline \multirow[t]{2}{*}{ Source de variation } & \multicolumn{2}{|c|}{ Production laitière } & \multicolumn{2}{|c|}{ Durée de lactation } & \multicolumn{2}{|c|}{ Intervalle entre vêlages } \\
\hline & DI & $\mathrm{Cm}$ & Dl & $\mathrm{Cm}$ & DI & $\mathrm{Cm}$ \\
\hline Races & 4 & 375048 * & 4 & 84443 & 3 & 2425 \\
\hline Saison de vêlage & 2 & 308595 & 2 & 10248 & 2 & 1090 \\
\hline Année de vêlage & 10 & 3487593 ** & 10 & $14819 * *$ & 11 & 23008 \\
\hline $\mathrm{N}^{\circ}$ de lactation & 7 & $541690 * *$ & 7 & 6354 & 6 & $46691^{* *}$ \\
\hline Race $x$ saison & 8 & 609528 & 8 & 6072 & 6 & 12402 \\
\hline Erreur & 243 & 430778 & 243 & 3998 & 99 & 16635 \\
\hline
\end{tabular}

$* \mathrm{P}<0,01 ; * * \mathrm{P}<0,05$ 


\section{Tableau II}

Production laitière, durée de lactation et intervalle entre vêlages en fonction de la race, de la saison de vêlage et du numéro de lactation

\begin{tabular}{|c|c|c|c|c|c|c|c|c|c|}
\hline \multirow[t]{2}{*}{ Facteurs de variation } & \multicolumn{3}{|c|}{$\begin{array}{c}\text { Production laitière } \\
\text { (litres) }\end{array}$} & \multicolumn{3}{|c|}{$\begin{array}{l}\text { Durée de lactation } \\
\text { (jours) }\end{array}$} & \multicolumn{3}{|c|}{$\begin{array}{c}\text { Intervalle entre vêlages } \\
\text { (jours) }\end{array}$} \\
\hline & $\mathbf{N} *$ & Moyenne & Ds $* *$ & $\mathbf{N}$ & Moyenne & Ds & $\mathbf{N}$ & Moyenne & Ds \\
\hline \multicolumn{10}{|l|}{ Races } \\
\hline $\mathrm{H}$ & 73 & $2508^{a}$ & 105 & 73 & 291 & 10 & 75 & 417 & 39 \\
\hline$J$ & 32 & 1818 bc & 137 & 32 & 251 & 13 & 17 & 435 & 67 \\
\hline $\mathrm{H} \times \mathrm{G}(\mathrm{F} 1)$ & 52 & $1940^{b}$ & 109 & 52 & 270 & 10 & - & - & - \\
\hline $\mathrm{J} \times \mathrm{zPb}(\mathrm{F} 1)$ & 57 & $1550^{d}$ & 106 & 57 & 257 & 10 & 22 & 412 & 53 \\
\hline $3 / 4 \mathrm{~J} \times \mathrm{zPb}$ & 61 & $1774^{\mathrm{c}}$ & 117 & 61 & 250 & 11 & 14 & 457 & 56 \\
\hline Moyenne générale & 275 & 1918 & 115 & 275 & 264 & 11 & 128 & 430 & 54 \\
\hline \multicolumn{10}{|l|}{ Saison de vêlage } \\
\hline Saison sèche & 88 & 1893 & 88 & 88 & 273 & 8 & 61 & 434 & 33 \\
\hline Pluies précoces & 78 & 1865 & 95 & 78 & 249 & 9 & 30 & 421 & 38 \\
\hline Pluies tardives & 109 & 1991 & 76 & 109 & 269 & 7 & 37 & 434 & 40 \\
\hline \multicolumn{10}{|l|}{ Année de vêlage } \\
\hline 1981 & 15 & 3369 & 217 & 15 & $334^{b}$ & 21 & & & \\
\hline 1982 & 12 & 2420 & 236 & 12 & $269 \mathrm{ab}$ & 23 & & & \\
\hline 1983 & 17 & 2062 & 208 & 17 & $297^{b}$ & 20 & & & \\
\hline 1984 & 33 & 2119 & 131 & 33 & $255^{a}$ & 13 & & & \\
\hline 1985 & 36 & 1839 & 122 & 36 & $220^{a}$ & 12 & & & \\
\hline 1986 & 23 & 1634 & 149 & 23 & $232^{a}$ & 14 & & & \\
\hline 1987 & 30 & 1529 & 130 & 30 & $248^{a}$ & 12 & & & \\
\hline 1988 & 21 & 1500 & 148 & 21 & $263 \mathrm{ab}$ & 15 & & & \\
\hline 1989 & 19 & 1584 & 168 & 19 & $282 \mathrm{ab}$ & 16 & & & \\
\hline 1990 & 26 & 1837 & 141 & 26 & $267 \mathrm{ab}$ & 14 & & & \\
\hline 1991 & 43 & 1204 & 118 & 43 & $263^{a}$ & 11 & & & \\
\hline Moyenne générale & 275 & 1918 & 160 & 275 & 264 & 16 & & & \\
\hline \multicolumn{10}{|l|}{$\mathbf{N}^{\circ}$ de la lactation } \\
\hline 1 & 42 & 1854 & 115 & 42 & 295 & 11 & 39 & 542 & 28 \\
\hline 2 & 52 & 1750 & 104 & 52 & 261 & 10 & 30 & 442 & 31 \\
\hline 3 & 46 & 1922 & 106 & 46 & 265 & 10 & 21 & 339 & 39 \\
\hline 4 & 42 & 2064 & 113 & 42 & 269 & 11 & 17 & 392 & 40 \\
\hline 5 & 36 & 2049 & 116 & 36 & 266 & 11 & 11 & 355 & 47 \\
\hline 6 & 26 & 1812 & 143 & 26 & 244 & 14 & 7 & 378 & 57 \\
\hline 7 & 14 & 1997 & 192 & 14 & 266 & 18 & 3 & 531 & 82 \\
\hline 8 & 17 & 1952 & 189 & 17 & 246 & 18 & - & - & - \\
\hline Moyenne générale & 275 & 1918 & 134 & 275 & 264 & 13 & 128 & 430 & 46 \\
\hline
\end{tabular}

* Nombre de lactations ; ** Déviation standard

$\mathrm{H}=$ Holstein $; \mathrm{J}=$ Jersiais $; \mathrm{G}=$ Goudali $; \mathrm{zPb}=$ zébu Peul blanc

a, b, c, d : les moyennes suivies de la même lettre sur la même colonne ne sont pas significativement différentes au seuil de 5 p. 100

\section{Effet de l'année de vêlage sur la production laitière}

Entre 1985 et 1990, la production laitière moyenne a été assez stable (entre 1500 et 1800 1), significativement inférieure à celle du début de l'essai (1981 et 1982) et supérieure à celle de la fin de l'étude. Ces différences de production semblent être liées à la structure du troupeau composée en majorité de Holstein en début de l'essai et de croisés Jersiais vers la fin de l'essai. Des fluctuations similaires liées à l'année sont signalées par Kiwuwa et coll. en Ethiopie (8). Ces auteurs imputent ces différences aux variations climatiques et à celles des effets zootechniques comme le numéro de lactation et les problèmes pathologiques.

\section{Effet du numéro de lactation sur la production laitière}

Le numéro de lactation a eu un effet important sur la production laitière. Généralement, la quantité de lait augmente avec les premiers veaux avant de diminuer avec les lactations de rang supérieur (16). Ici, la faible production des premières lactations a semblé être compensée par la prédominance des vaches Holstein dans le troupeau au début de l'essai. Kiwuwa et coll. (8) ont également trouvé une influence significative des numéros de lactation sur la production laitière. Ils ont ainsi obtenu 1 724, 1892,1988 et 1883 litres respectivement à la $1^{\mathrm{re}}, 2^{\mathrm{e}}, 3^{\mathrm{e}}$ et $4^{\mathrm{e}}$ lactation pour leur essai, la même race étant utilisée sur plusieurs années. 


\section{Effet de la durée de lactation sur la production laitière}

L'analyse de variance a montré une différence significative $(\mathrm{P}<$ $0,05)$ entre les années pour ce paramètre. En revanche, aucune différence significative $(\mathrm{P}>0,05)$ n'a été observée entre les races, les saisons de vêlage et les numéros de lactation. Les durées de lactation moyennes sont présentées dans le tableau II en fonction de la race, de la saison, de l'année de vêlage et du numéro de lactation.

- Effet de la race sur la durée de lactation

Sans que la différence ait été significative, la durée de lactation des vaches Holstein en race pure (291 jours) et en croisement (270 jours) a été supérieure à celle des autres types génétiques. Si la durée de lactation de la Jersiaise pure a été de 251 jours, celle de son croisement avec le zébu a augmenté à la F1 (257 jours), mais cet avantage a été perdu avec la 3/4 Jersiaise (250 jours). La variabilité génétique trouvée ici est signalée par plusieurs auteurs $(2,4$, $5,8,9)$. Les conditions d'élevage, les facteurs climatiques et zootechniques sont signalés comme responsables de cette variation.

\section{- Effet de la saison de vêlage sur la durée de lactation}

Les vaches qui ont vêlé en saison sèche ont eu tendance à avoir une durée de lactation (273 jours) supérieure à celles qui ont vêlé en deuxième moitié de la saison des pluies (269 jours) et en première moitié de la saison des pluies (249 jours). Mais, ces différences n'ont pas été significatives au seuil de 5 p. 100. La disponibilité alimentaire tout au long de l'année pouvait être à l'origine de ces résultats. La durée moyenne de lactation (264 jours) a été inférieure aux 350 jours rapportés par Kiwuwa et coll. en Ethiopie (8).

- Effet de l'année de vêlage sur la durée de lactation

La durée de lactation a varié significativement $(\mathrm{P}<0,05)$ de 1981 à 1991. La variation annuelle de la composition génétique du troupeau expliquerait la fluctuation observée. La même variation a été signalée par Kiwuwa et coll. en Ethiopie (8).

\section{- Effet du numéro de lactation sur la durée de lactation}

Le numéro de lactation a affecté de manière significative $(\mathrm{P}<$ $0,05)$ la durée de lactation. De la première à la huitième lactation, les durées moyennes de lactation ont été respectivement de 295, 261, 265, 269, 244, 266 et 246 jours. Ces résultats ont montré que la durée de lactation a varié avec le numéro de lactation. En Ethiopie, Kiwuwa et coll. (8) ont également obtenu des résultats significatifs $\left(406,395,332\right.$ et 305 jours respectivement en $1^{\mathrm{re}}, 2^{\mathrm{e}}, 3^{\mathrm{e}}$ et $4^{\mathrm{e}}$ lactation). Ces auteurs supposent que la durée de lactation décroît lorsque le numéro de lactation augmente ; elle serait liée au vieillissement de la vache.

\section{Intervalle entre vêlages}

L'analyse de variance (tableau I) a montré une différence significative $(\mathrm{P}<0,05)$ entre les numéros de lactation pour ce paramètre. Par ailleurs, l'analyse de variance n'a pas relevé de différences significatives $(P>0,05)$ entre les races et les saisons. L'intervalle moyen entre vêlages est présenté au tableau II en fonction des groupes génétiques, de la saison de vêlage et du numéro de lactation.

\section{Effet de la race sur l'intervalle entre vêlages}

La race n'a pas influencé significativement l'intervalle entre vêlages en général de 430 jours avec une variation de 412 à 457 jours. Il a été plus long chez la race pure Jersaise (435 jours) que chez la Holstein (417 jours) et s'est allongé avec le niveau de sang Jersiais. Ce résultat suppose que l'intervalle entre vêlages n'est pas génétiquement influencé et serait beaucoup plus affecté par les facteurs environnementaux. Par conséquent, l'amélioration de ce paramètre dépend de l'amélioration des conditions d'élevage. Madalena et coll. (10) et Parmar et coll. (15) ont obtenu le même résultat et ont montré que l'intervalle entre vêlages était influencé par l'âge des vaches. Meyn et Wilkins (14) et Wijeratne (20) ont confirmé ces résultats en montrant que l'intervalle de mise bas diminuait avec l'âge de la vache et, de plus, que l'intervalle s'allongeait après la mise bas d'un veau mâle et, donc, était plus court après la mise bas d'une femelle.

\section{Effet de la saison de vêlage sur l'intervalle entre vêlages}

La saison de vêlage n'a pas eu d'effets importants sur l'intervalle entre vêlages. Plus court en première moitié de la saison des pluies (421 jours), l'intervalle entre vêlages a été le même en saison sèche et en deuxième moitié de la saison des pluies (434 jours). La supplémentation alimentaire pendant la saison sèche aurait permis de surmonter les différences saisonnières susceptibles d'affecter l'effet de la saison de vêlage sur l'intervalle entre les vêlages. Veisseyre (19) et Choisis et coll. (3) ont plutôt trouvé des effets saisonniers significatifs sur l'intervalle entre vêlages. Il est probable que les conditions alimentaires des différentes saisons étaient plus ou moins défavorables.

\section{Effet du numéro de lactation sur l'intervalle entre vêlages}

L'intervalle entre vêlages de la première à la septième lactation a varié significativement de 355 à 542 jours avec une moyenne générale de 430 jours, comme cela a été rapporté par d'autre auteurs à la Jamaïque $(17,18)$, en Inde (15) et au Brésil (10), c'està-dire que l'intervalle entre vêlages augmente avec l'âge des vaches, les vaches âgées ayant tendance à avoir un intervalle plus long.

\section{- CONCLUSION}

Les résultats de cette étude ont montré que la production laitière des races pures Holstein et Jersiaise, sans être comparable à celle des pays d'où elles sont originaires, a été significativement supérieure à celle des autres types génétiques étudiés, la Holstein ayant été plus performante que la Jersiaise. Par ailleurs, en croisement avec le zébu local, la race Holstein a été significativement plus performante que la race Jersiaise en quantité de lait produite. Enfin, la production laitière des vaches croisées Jersiaises a augmenté avec le niveau de substitution par le zébu local.

Parmi les facteurs étudiés, la saison de vêlage n'a pas significativement affecté la production laitière. En revanche, l'année de vêlage et le numéro de lactation ont significativement influencé la production laitière et la durée de lactation. Cependant, l'utilisation de ces différents types génétiques dans les hauts plateaux de l'ouest du Cameroun suppose une amélioration préalable des conditions d'élevage (habitat, hygiène), des conditions favorables d'alimentation et une bonne organisation de l'élevage qui ont été possibles en station.

Il est à noter que les races pures qui permettent d'obtenir une meilleure production laitière sont souvent très exigeantes en matière d'entretien et que le coût de cet entretien annulerait les différences réalisées. En revanche, les races croisées associant la rusticité et une production moyenne pourraient être plus rentables. Mais ici se pose encore le problème de la gestion du troupeau (organisation de l'élevage), surtout si l'on envisage le passage en milieu éleveur. L'analyse financière de la production laitière ainsi que la composition du lait des différentes races et de leurs croisements dans les conditions des hauts plateaux de l'ouest du Cameroun constituent une nécessité. 


\section{Remerciements}

Les auteurs sont très reconnaissants à l'Institut de recherches agricoles pour le développement (Irad) qui a financé ce programme de recherche à Bambui (Cameroun). Des remerciements sont également adressés aux bergers, aux techniciens et à tous les travailleurs du Centre de recherche de Bambui.

\section{BIBLIOGRAPHIE}

1. ADENIJI K.O., 1985. Livestock breding in Africa. World Rev. Anim. Prod., 21: 63-73.

2. BRANTON C., MCDOWELL R.E., BROWN M.A., 1966. Zebu European crossbreeding as a basis of cattle improvement in the USA Baton Rouge, LA, USA, Louisiana Agriculture Experimental Station, p. 39. (Southern Cooperative Series, Bull. No. 114)

3. CHOISIS I.P., CERVANTES N., LHOSTE P., 1990. Effets saisonniers sur certains paramètres de la production bovine dans les élevages mixtes de l'état de Colima au Mexique. Revue Elev. Méd. vét. Pays trop., 43 : 97-104

4. DUC N.V., TANEJA V.K., 1984. Comparative performance of pure breed and crossbreed grades in India. Indian J. Anim. Sci., 54 1023-1028.

5. FRIESTAS A.F., MADALENA F.E., MARTINEZ M.L., 1980. Age at calving intervals of Holstein-Friesian and crossbreed Holstein-Friesian: Gir (translated title). Pesq. Agropec. Bras., 15: 101-105.

6. IEMVT, 1988. Résultats du contrôle laitier des espèces bovine et caprine. Maisons-Alfort, France, lemvt, 56 p.

7. KAMGA P., KAMGA B., AWAH N., 1989. Production of grass silage at Bambui: techniques, chemical and bacteriological composition. In Actes du seminaire régional sur les fourrages et l'alimentation des ruminants, N'Gaoundere, Cameroun, 16-20 novembre 1987 (résumés). Maisons-Alfort, France, lemvt, p. 108-109. (Etudes et synthèses de I'lemvt $\left.n^{\circ} 30\right)$

8. KIWUWA G.H. TRAIL J.C.M KURTU M.Y, WORKU G. ANDERSON F.M., DURKIN J., 1983. Crossbreed dairy cattle productivity in Arsi Region, Ethiopia. Addis Ababa, Ethiopia, ILCA, p. 1-29. (Research Report No. 11)

9. LETENNEUR L., 1978. Crossbreeding N'Dama and Jersey cattle in Ivory Coast. World Anim. Rev., 27: 36-42.
10. MADALENA F.E, FREITAS A.F MARTINEZ M.L., 1978. Comparative evaluation of milk production in Holstein-Friesian and Holstein-Fresian x Gir cows. In: Proc. 4th World Conf. on Animal Production, Buenos Aires, Argentina, 20-26 August 1978, vol. 2, p. 650-658.

11. MBAH D.A., 1981. Note sur l'influence des saisons sur la production laitière à Wakwa. Revue sci. tech. Off. int. Epizoot., 1 : 145-148.

12. MBAH D.A., MBANYA J., MESSINE O., 1987. Performance of Holsteins, Jerseys and their zebu crosses in Cameroon: Preliminary results. Sci. Tech. Rev., 3: 116-126.

13. MEYER C., DENIS J.P., 1999. Elevage de la vache laitière en zone tropicale. Montpellier, France, Cirad, 314 p.

14. MEYN K., WILKINS J.V., 1974. Breeding for milk in Kenya, with particular reference to Sahiwal Stud. World Anim. Rev., 11: 24-30.

15. PARMAR O.S., DEV D.S., DHAR N.L., 1980. Interse mating among Jersey and Hariana cattle. Indian J. Dairy Sci., 33: 465-467.

16. POLY J., VISSAC B., 1958. L'incidence des variations d'intervalles de vêlage sur la production de la vache laitière. Le lait, 38 : 598-606.

17. PRADA N., 1979. Dairy crossbreeding programme in Cuba. In: colloq. Value of Crossbreeding in Different Production Systems, Havana, Cuba, 23-29 September 1979. In Memoria Assoc. Latinamericana Prod. Anim., 14: 163-167.

18. RUBIO R., 1976. Ganado Costeno con cuernos. Man. Asist. tec., 21: 83-106.

19. VEISSEYRE R., 1975. Technologie du lait, constitution, récolte, traitement et transformation du lait, $3^{\mathrm{e}}$ éd. Paris, France, La maison Rustique, $714 \mathrm{p}$.

20. WIJERATNE W.V.S., 1970. Crossbreeding Sinhala cattle with Jersey and Friesian in Ceylon. Anim. Prod., 12: 473-483.

21. WILKINS J.V., 1984. Criollo cattle of America. Anim. Genet. Res. Inf., 1: 1-19.

22. WORLD-WIDE SIRES, 1996. Holstein bulls records. Hanford, CA, USA, p. 2.

Reçu le 23.11.1999, accepté le 26.07.2001 


\section{Summary}

Kamga P., Mbanya J.N., Awah N.R., Mbohou Y., Manjeli Y., Nguemdjom A., Kamga Pamela B., Njwe R.M., Bayemi P.H., Ndi C1., Imélé H., Kameni A. Effect of the Calving Season and Zootechnical Parameters on Milk Yield in the Western Highlands of Cameroon

Data on 275 lactations were collected and analyzed between 1981 and 1991 in Holstein and Jersey breeds and their crosses with Gudali and White Fulani zebus in the western highlands of Cameroon. The objective of the study was to evaluate the effect of the calving season, calving intervals and other zootechnical parameters on milk yield. The following results were obtained:

- Milk yield of the Holstein ( $=73$ ) and Jersey ( $=32)$, although lower than that obtained in their country of origin, was significantly higher than that of the genetic types studied, with the Holstein producing more milk than the Jersey (2508 \pm 105 I in $291 \pm 10$ days vs $1818 \pm 137$ I in $251 \pm 13$ days, respectively);

- In first generation crosses with the local zebu, the Holstein $(n=52)$ produced significantly more milk than the Jersey $(n=$ 57). The Holstein $x$ zebu Gudali produced more milk than the Jersey $x$ White Fulani $(1940 \pm 109$ | in $270 \pm 10$ days vs 1550 \pm 106 I in $257 \pm 10$ days, respectively). Milk yield of 3/4 Jersey crossbreds $(n=61)$ was higher $(1774 \pm 117$ I in $250 \pm 11$ days);

- While the calving year and lactation number significantly affected milk yield, the calving season did not.

Investigations on financial profitability and milk composition should be carried out for concrete recommendations.

Key words: Cattle - Crossbreeding - Milk production - Parturition interval - Site factor - Lactation number - Cameroon.

\section{Resumen}

Kamga P., Mbanya J.N., Awah N.R., Mbohou Y., Manjeli Y., Nguemdjom A., Kamga Pamela B., Njwe R.M., Bayemi P.H., Ndi C1., Imélé H., Kameni A. Efectos de la época de parto y de algunos parámetros zootécnicos sobre la producción láctea en las mesetas altas al oeste de Camerún

Entre 1981 y 1991, se colectaron y analizaron datos sobre 275 lactancias. El objetivo del presente trabajo fue el de estudiar el efecto de la época de parto y de algunos parámetros zootécnicos sobre la producción láctea y el intervalo entre partos de las razas Holstein y Jersey y de los cruces respectivos con cebú Gudali y cebú Fulani blanco en las mesetas altas al oeste de Camerún. Los resultados demuestran lo siguiente:

- la producción lechera de las razas puras Holstein $(n=73)$ y Jersey ( $\mathrm{n}=32$ ), aunque no comparable con aquella obtenida en los países de origen, fue significativamente superior a la de otros tipos genéticos estudiados, siendo el rendimiento de la Holstein superior al de la Jersey: $2508 \pm 105$ I contra $1818 \pm$ 137 I, durante $291 \pm 10$ o $251 \pm 13$ días;

- en cruces de primera generación con el cebú local, la Holstein $(n=52)$ produjo significativamente más leche que la Jersey $(\mathrm{n}=57)$; la Holstein $\mathrm{x}$ cebú Gudali produjo más leche que la Jersey x cebú Fulani blanco: $1940 \pm 109$ I, durante 270 \pm 10 días, contra $1550 \pm 106 \mathrm{l}$, durante $257 \pm 10$ días. La producción lechera de los cruces tres cuartos Jersey fue superior $(n=61): 1774 \pm 117$ durante $250 \pm 11$ días;

- el año y el numero de lactacia influenciaron significativamente la producción lechera y la duración de la lactación, mientras que la época de parto no tuvo efecto significativo sobre estos dos parámetros.

Las investigaciones sobre el análisis financiero y la composición de la leche deberían seguir para proporcionar recomendaciones más concretas.

Palabras clave: Ganado bovino - Cruzamiento - Producción lechera - Intervalo entre partos - Caracteristica del sitio Numero de lactacia - Camerún. 